\title{
CONTRIBUCIÓN A LA FLORA VASCULAR Y VEGETACIÓN DE LOS VALLES SECOS INTERANDINOS DE LOS RÍOS TOROBAMBA (AYACUCHO) Y PAMPAS (APURÍMAC), SUR DEL PERÚ
}

\section{CONTRIBUTION TO THE VASCULAR FLORA AND VEGETATION FROM TOROBAMBA (AYACUCHO) AND PAMPAS (APURÍMAC) INTER-ANDEAN DRY VALLEYS, SOUTHERN PERU}

Fiorella Eduardo-Palomino ${ }^{1}$, Héctor Chuquillanqui ${ }^{2}$, Pablo Najarro ${ }^{3}$ y Reynaldo Linares-Palomino ${ }^{4}$

\section{Resumen}

Presentamos una primera aproximación a la flora vascular y vegetación de los valles secos de los ríos Torobamba y Pampas ubicados en Ayacucho y Apurímac, Perú. Usando Parcelas Modificadas intensivas Whittaker y muestreos asistemáticos registramos 91 especies y morfoespecies de plantas vasculares, cinco de las cuales son endémicas y cuatro están listadas como flora silvestre amenazada según la legislación actual. Asteraceae y Poaceae fueron las familias mejor representadas ( $14.3 \%$ y $13 \%$ de todas las especies, respectivamente). La forma de vida predominante fueron las hierbas $(60 \%)$. Registramos 60 especies en las parcelas del río Pampas, mientras que en las del Torobamba fueron 63 especies. La especie más abundante y dominante fue Eriotheca cf. vargasii (Malvaceae, 49\% - 63\% de todos los individuos en las parcelas), especie endémica de estos valles. El núcleo más diverso parece encontrarse en el mismo valle del Apurímac. Aun así, los bosques de los valles ubicados en zonas más altas y alejadas de este núcleo de diversidad no son necesariamente un subconjunto florístico. Los parches de bosques de los valles interandinos del Pampas y Torobamba sólo comparten entre una a tres especies con aquellos del Apurímac, sugiriendo altos valores de diversidad beta. Por lo tanto, para conservar adecuadamente estos bosques secos no será suficiente con sólo proteger la zona más diversa. Por el contrario, un corredor de áreas de conservación que conecte parches de bosque a lo largo de todo el gradiente altitudinal parece ser la mejor opción.

Palabras clave: bosque seco interandino, endemismo, inventarios cuantitativos, parcelas, relaciones florísticas, riqueza de especies.

\begin{abstract}
We present a first approximation to vegetation and vascular flora from the Torobamba and Pampas dry valleys in Ayacucho and Apurímac, southern Peru. We used intensive modified Whittaker plots and nonsystematic surveys to record 91 vascular plant species and morph species. Five of these are endemic and four are considered as threatened in the current Peruvian legislation. Asteraceae and Poaceae were the most representative families $(14.3 \%$ and $13 \%$ of all species, respectively). The predominant life-form was herbs $(60 \%)$. We recorded 60 species at the Pampas river valley plots and 63 species at the Torobamba plots. The most abundant and dominant species was Eriotheca cf. vargasii (Malvaceae, $49 \%$ - 63\% of all individuals in the plots), an endemic species of these southern valleys. Our data suggest that the most diverse area is located at the Apurimac valley. Even so, the forest patches located at higher elevations and away from this diversity center are not necessarily a floristic subset. The dry forest patches in the Pampas and Torobamba inter-Andean valleys share only one to three species with those at Apurimac, suggesting high beta diversity values. Therefore, in order to conserve these dry forests focusing in the protection of the most diverse zone seems not to be enough. Rather a conservation corridor connecting forest patches along the entire altitudinal gradient seems to be the best option.

Key words: endemism, floristic relationships, inter-Andean dry forest, species richness, plots, quantitative inventories.
\end{abstract}




\section{Introducción.}

El bosque tropical estacionalmente seco (BTES) está probablemente entre los biomas más amenazados del Neotrópico (Miles et al., 2006; Portillo-Quintero \& Sánchez-Azofeifa, 2010). Históricamente, los esfuerzos de investigación científica realizados en ellos son escasos. Además, existen pocas áreas de conservación específicamente designadas para protegerlos cuando se comparan con otros biomas, especialmente aquellos en regiones más húmedas (Sánchez-Azofeifa et al., 2005). Los BTES en el Perú se encuentran principalmente en la región de la costa noroeste y sus estribaciones andinas correspondientes. Adicionalmente, hay áreas importantes al sur de Tarapoto (departamento de San Martín) y en los bosques secos inter-andinos (BSI) ubicados en los valles de los ríos Marañón, Mantaro y Apurímac (Linares-Palomino, 2004, 2006). En los últimos diez años se ha generado valiosa información sobre los BSI (Särkinen et al., 2011; Särkinen et al., 2012; Gagnon et al., 2015; Marcelo-Peña et al., 2015); no obstante, aún se conoce poco sobre la biología de estos bosques secos, tanto en términos descriptivos (florísticos) como funcionales (ecológicos).

Los BSI del sistema del Apurímac, que incluyen entre otros los valles del Pampas y el Pachachaca, son probablemente los menos conocidos. Aun cuando Augusto Weberbauer (1945) reporta haber pasado por estos bosques, los primeros estudios florísticos integrales y detallados fueron hechos por el botánico cusqueño César Vargas (1949). Posteriormente, Werner Rauh (1958) recorrió y describió la vegetación de los valles del Apurímac y del Pachachaca, enfocándose principalmente en las cactáceas. Desde entonces, y hasta años recientes, son pocos los estudios que han contribuido al conocimiento de la vegetación de estos bosques (Aréstegui et al., 2012; Huamantupa \& Cuba, 2013; Linares-Palomino, 2013).

Nuestro conocimiento florístico del sistema de BSI del Apurímac está principalmente basado en estudios realizados en el mismo valle del Apurímac. Sin embargo, los BSI en esta región se extienden a todos los tributarios de este río, entre los 1000 y $2900 \mathrm{msnm}$ (Linares-Palomino, 2013). Ante este escenario de información escasa para un ecosistema con altos niveles de fragmentación y amenazado globalmente (DRYFLOR et al., 2016), nuestro estudio evaluó la situación actual, en términos de diversidad, composición y estructura vegetal de los BSI de valles alejados del río Apurímac (ríos Torobamba y Pampas). En base a estos datos analizamos las relaciones florísticas de estos valles con otros parches de bosques secos en el sistema de valles del Apurímac para identificar subgrupos vegetacionales que tendrían su origen en el patrón ecológico y biogeográfico denominado decaimiento de la similitud de comunidades con la distancia geográfica (Nekola \&
White, 1999; Morlon et al., 2008). Este patrón biogeográfico aplicado a nuestra área de estudio nos sugiere que el valle del Apurímac conformaría el centro de diversidad del sistema de BSI del Apurímac, y que parches de BSI alejados y a mayor altitud de este núcleo central serían más paupérrimos en términos de diversidad. Los objetivos de este trabajo fueron 1) describir la vegetación de parches de bosques seco interandino poco conocidos, 2) evaluar si los bosques del sistema de valles secos del Apurímac constituyen una unidad homogénea o si existen subgrupos vegetacionales y finalmente 3) evaluar el estado de conservación de estos bosques para proponer acciones necesarias para su manejo.

\section{Métodos.}

La expedición se realizó hacia el final de la temporada seca, entre el 29 de octubre y el 3 de noviembre del 2014 y evaluamos ocho puntos de monitoreo entre los 1800 y $2400 \mathrm{msnm}$. Tres en el valle del río Pampas y cinco en el valle del río Torobamba (Tabla 1 y Figura 1). El valle del río Pampas a la altura de Río Blanco (2093 msnm, Chincheros, Apurímac) tiene una precipitación de $611 \mathrm{~mm}$ anuales, concentrando $407 \mathrm{~mm}$ (https://en.climatedata.org/location/876262/) entre diciembre y marzo. El valle del río Torobamba a la altura de San Miguel (2612 msnm, La Mar, Ayacucho) tiene una precipitación de $536 \mathrm{~mm}$ anuales, concentrando $352 \mathrm{~mm}$ (https://en.climate-data.org/location/876274/) entre diciembre y marzo.

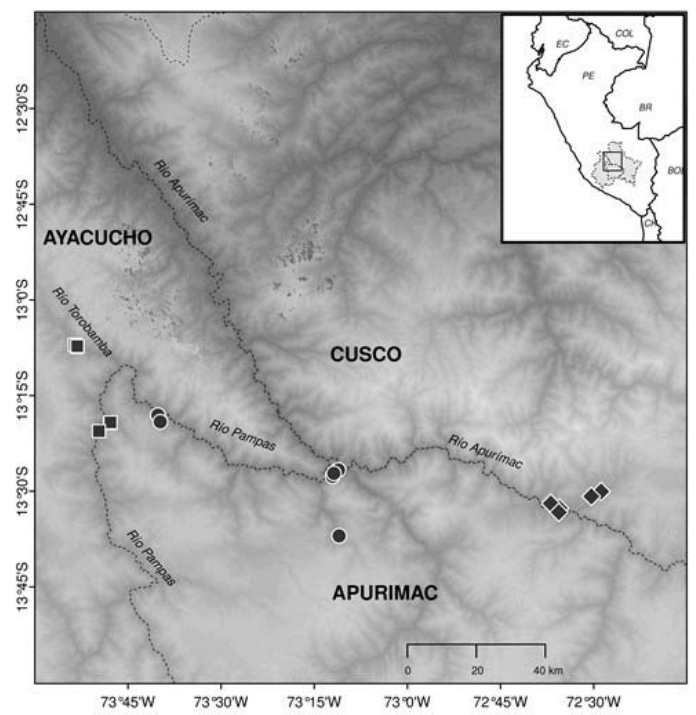

Figura 1. Localización de las parcelas evaluadas. Cuadrados: izquierda parcelas del valle del río Torobamba, derecha parcelas del río Pampas. Redondos: parcelas evaluadas por K. Dexter (2012, datos no publicados). Rombos: parcelas evaluadas por Aréstegui et al. (2012). 
Evaluamos la flora vascular utilizando parcelas Modificadas Intensivas Whittaker (PMW; Barnett \& Stohlgren, 2003). Para cada PMW delimitamos en cada punto de monitoreo un área de $20 \mathrm{~m}$ x $20 \mathrm{~m}$ donde registramos el diámetro a la altura de pecho (DAP) de todas las plantas leñosas y cactáceas erectas con al menos $5 \mathrm{~cm}$ de DAP. Dentro de cada parcela ubicamos una subparcela central de $20 \mathrm{~m} \times 5 \mathrm{~m}$ donde registramos todos los arbustos y cactáceas erectas con DAP menor a $5 \mathrm{~cm}$ y $1.5 \mathrm{~m}$ de altura. En el centro de esta subparcela anidamos una siguiente subparcela de $2 \mathrm{~m}$ x $5 \mathrm{~m}$ y registramos todas las plantas presentes, incluyendo hierbas y cactáceas rastreras y globulares. Adicionalmente, registramos visualmente la cobertura de especies de arbustos menores, herbáceas y cactáceas de porte pequeño en cuatro subparcelas de $2 \mathrm{~m} \times 0.5 \mathrm{~m}$, ubicadas en los vértices de la parcela de $20 \mathrm{~m}$ x $20 \mathrm{~m}$ considerando la proyección horizontal de la parte aérea sobre el suelo, donde una superficie de $10 \times 10 \mathrm{~cm}$. representó $1 \%$ de cobertura. Finalmente, con el fin de obtener una muestra representativa de la flora aledaña a cada parcela, colectamos y tomamos fotografías de toda especie adicional encontrada fuera de las parcelas a través de recorridos asistemáticos.

La identificación de las especies se hizo en campo cuando se trató de especies fácilmente identificables por los evaluadores, utilizando las guías de plantas vasculares de los BSI del Torobamba (LinaresPalomino, 2016). Los individuos que no se identificaron directamente fueron fotografiados $y$ colectados para su tratamiento e identificación en el herbario USM (Museo de Historia Natural, Universidad Nacional Mayor de San Marcos).
En base a la información de las PMW, se evaluó y comparó la riqueza específica mediante curvas de acumulación de especies y estimadores noparamétricos (Chao, Jackknife1, Jackknife2, y Bootstrap). En base a todas las especies registradas, en parcelas de $400 \mathrm{~m}^{2}$, se calculó la riqueza esperada mediante estimadores no-paramétricos. Tanto para el valle del Pampas y Torobomba, se graficaron de manera independiente curvas de acumulación de especies que incluyeron las subparcelas $\left(1 \mathrm{~m}^{2}, 10 \mathrm{~m}^{2} \mathrm{y}\right.$ $100 \mathrm{~m}^{2}$ ) y la parcela completa $\left(400 \mathrm{~m}^{2}\right)$. A su vez, se evaluó el número de especies potencialmente presentes en cada valle mediante estimadores no-paramétricos en base a las subparcelas de $1 \mathrm{~m}^{2}$, dado que el método requiere unidades muestrales de tamaño fijo.

Por último, exploramos las relaciones florísticas y estructurales entre los diferentes parches evaluados en este estudio, y con datos de otras evaluaciones disponibles para el sistema de BSI del Apurímac (K. Dexter datos no publicados; Aréstegui et al., 2012). Se empleó el índice de disimilaridad de Bray-Curtis, como un proxi de la diversidad beta entre parcelas, en un análisis de clasificación (UPGMA). Todos los análisis se hicieron usando el paquete vegan (Oksanen et al., 2016) en el ambiente de computación R (R Core Team, 2014), excepto los cálculos del índice de diversidad de Shannon $\left(\mathrm{H}^{\prime}=\sum p_{i} \ln p_{i}\right.$, Magurran, 2004). Estos se hicieron para cada parcela evaluada utilizando el programa PAST (Hammer et al., 2001).

Tabla 1. Coordenadas (UTM, Zona 18L) y altitud de las parcelas evaluadas. Se incluyen las parcelas de K. Dexter, 2012 (datos no publicados, Apu 55, 56, 57, 58, 59 y 60) y Aréstegui et al., 2012 (Wayronqa, Cunyaq, Qonoq, Sauceda y Wayna).

\begin{tabular}{llllcr}
\hline Valle & ID & UTM1 & UTM2 & $\begin{array}{c}\text { Altitud } \\
\text { (msnm) }\end{array}$ & Fuente $^{*}$ \\
\hline Pampas & Pam1 & 630214 & 8526928 & 1940 & 1 \\
Pampas & Pam2 & 630307 & 8527148 & 2090 & 1 \\
Pampas & Pam3 & 626913 & 8524642 & 1845 & 1 \\
Torobamba & Tor1 & 620639 & 8549142 & 2258 & 1 \\
Torobamba & Tor2 & 620026 & 8549636 & 2334 & 1 \\
Torobamba & Tor3 & 620679 & 8549030 & 2331 & 1 \\
Torobamba & Tor4 & 620744 & 8549166 & 2235 & 1 \\
Torobamba & Tor5 & 620743 & 8549226 & 2195 & 1 \\
Apurímac & Apu55 & 644101.9 & 8529171.6 & 1900 & 2 \\
Apurímac & Apu56 & 644774.7 & 8527243.8 & 2950 & 2 \\
Apurímac & Apu57 & 696533.6 & 8493934.1 & 2600 & 2 \\
Apurímac & Apu58 & 694760.3 & 8511158.8 & 2200 & 2 \\
Apurímac & Apu59 & 696494.5 & 8512990.2 & 1040 & 2 \\
Apurímac & Apu60 & 695139.5 & 8512004.4 & 1750 & 2 \\
Apurímac & Cunyaq & 760613 & 8501413.9 & 1980 & 3 \\
Apurímac & Qonoq & 758112.2 & 8502776.4 & 2150 & 3 \\
Blanco & Wayronqa & 772780.55 & 8506089.4 & 2850 & 3 \\
Blanco & Sauceda & 769935.34 & 8504712.26 & 2420 & 3 \\
Apurímac & Wayna & 760438.91 & 8500214.63 & 2200 & 3 \\
\hline * Este estudio; $:$ Dexter & datos no publicados; $3:$ Aréstegui & \\
\hline
\end{tabular}

* 1: Este estudio; 2: Dexter, datos no publicados; 3: Aréstegui et al., 2012. 


\section{Resultados.}

Diversidad y endemismo en los BSI del Pampas y Torobamba.

Registramos 91 especies y morfoespecies de plantas vasculares, de las cuales logramos identificar 81,8 taxa sólo a nivel de familia, 29 sólo a nivel de género y 44 a nivel de especie (Anexo 1). Diez morfoespecies no presentaron estructuras fértiles que ayudaran en su determinación como consecuencia de realizar los inventarios durante la temporada seca y no fueron identificadas. Logramos identificar completamente a 44 especies, agrupadas en 42 géneros y 19 familias botánicas. Respecto a las familias con especies identificadas, 15 pertenecieron a la clase Magnoliopsida, 3 a la clase Liliopsida y una a la clase Polypodiopsida (helechos).

Sólo cinco especies, Tillandsia latifolia Meyen (Bromeliaceae), Heliotropium pilosum Ruiz \& Pav. (Boraginaceae), Browningia viridis (Rauh\&Backeb.) Buxb. (Cactaceae), Coursetia fruticosa (Cav.) J. F. Macbr. (Fabaceae) y Eriotheca cf. vargasii (Malvaceae) son reconocidas como endémicas. Otras cuatro especies adicionales están listadas categorizadas como Especies Amenazadas de Flora Silvestre (Decreto Supremo 043-2006-AG): Vasconcellea quercifolia A. St.-Hil. (Caricaceae), en Peligro Crítico; Tecoma fulva (Cav.) G. Don (Bignoniaceae), Cyathostegia mathewsii (Benth.) Schery (Leguminosae) y Argyrochosma nivea (Poir.) Windham (Pteridaceae), como Vulnerables. Dos especies se encuentran en la Lista Roja de Especies Amenazadas de la Unión Internacional para la Conservación de la Naturaleza (UICN, 2013): Coursetia fruticosa considerada de preocupación menor (LC) y Eriotheca cf. vargasii como especie no evaluada para ninguna de las categorías (NE). Además, seis especies, Opuntia ficus-indica (L.) Mill., Opuntia quitensis F.A.C.Weber, Austrocylindropuntia subulata (Muehlenpf.) Backeb., Cylindropuntia tunicata (Lehm.) F. M. Knuth, Browningia viridis $\mathrm{y}$ Cleistocactus sp., todas ellas Cactaceae, se mencionan en el Anexo II de la Convención sobre el Comercio Internacional de Especies Amenazadas de Fauna y Flora Silvestres (UNEP-WCMC, 2015).

Dentro de la clase Liliópsida se registraron siete familias, dentro de la clase Magnoliópsida veintidós familias y dentro de la clase Polypodiópsida una familia. La familia Asteraceae es la más representativa en la zona con un $14.3 \%$ de las especies y morfoespecies (4 especies y 9 morfoespecies), seguida de Poaceae con un 13\% (10 especies y 2 morfoespecies). Las familias Cactaceae y Fabaceae representan cada una un $6.6 \%$ de todas las especies, Euphorbiaceae y Sapindaceae un $5.5 \%$, Malvaceae $4.4 \%$, Bromeliaceae un $3.3 \%$ y las demás familias menos de $2 \%$.

La forma de vida predominante son las hierbas (58 especies y morfoespecies), que representan más del
$60 \%$ de todas las especies y morfoespecies registradas (63.7\%). Los arbustos están representados por 18 especies y morfoespecies (19.7\%). Tanto los árboles como las suculentas (incluyendo cactus rastreros, globulares y erectos o arborescentes) están representados con $9(9.9 \%)$ y $6(6.6 \%)$ especies, respectivamente.

Los estimadores de riqueza no-paramétricos muestran valores contrastantes en el número potencial de especies. Según el estimado más conservador (Bootstrap), 14 especies no han sido detectadas; mientras que según la estimación más extrema (Jackknife 2), se indican 52 especies de plantas vasculares por descubrir en estos bosques (Figura 2). Diversidad y estructura de los BSI del Pampas.

En las PMW se registraron 60 especies y morfoespecies pertenecientes a 23 familias. Cinco especies corresponden a los árboles Vachellia aroma, Cyathostegia mathewsii, Cnidoscolus urens, Eriotheca cf. vargasii y Dodonaea viscosa, a las que podemos sumar por su alto crecimiento a Browningia viridis, que se encuentra dentro de las cuatro suculentas registradas. Además, se registraron 9 arbustos, 35 hierbas, 4 helechos y 3 epífitas.

Las familias más dominantes fueron Asteraceae y Poaceae ambas con 7 especies y morfoespecies. Se registraron 128 individuos con un DAP $\geq 5 \mathrm{~cm}$ (incluye individuos de tallos múltiples) en las parcelas de 400 $\mathrm{m}^{2}$, de los cuales se identificaron cuatro especies leñosas y suculentas. Estas son en orden decreciente: Eriotheca cf. vargasii (63 individuos), Vachellia aroma (62 individuos), Cnidoscolus urens (2 individuos) y Browningia viridis (1 individuo). La especie con mayor DAP en las parcelas de $400 \mathrm{~m}^{2}$ fue Eriotheca cf. vargasii $(50.5 \mathrm{~cm}$, en la parcela Pam 3$)$. En las sub parcelas de $1 \mathrm{~m}^{2}$ y parcelas de $10 \mathrm{~m}^{2}$, la especie dominante fue Serjania squarrosa. Las especies que registraron mayor cobertura (5\%) en las sub parcelas de $1 \mathrm{~m}^{2}$ fueron Heteropogon contortus, Trixis sp. y Serjania squarrosa.

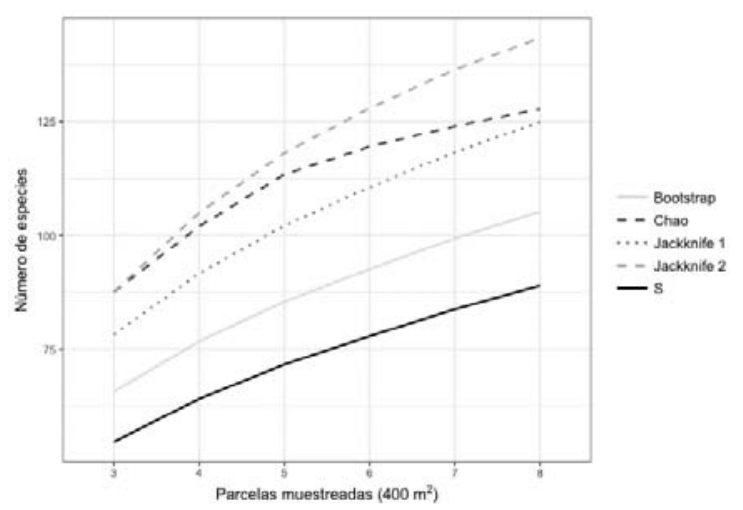

Figura 2. Riqueza de especies esperada en los valles del río Pampas y Torobamba usando cuatro estimadores. S: riqueza de especies observada. 
Las curvas de acumulación de especies obtenidas a partir de las tres parcelas evaluadas en el valle del río Pampas muestran que en las parcelas de $400 \mathrm{~m}^{2}$ el número máximo de especies registradas fue de 36 , muy cercano a las 35 especies registradas en parcelas de 100 $\mathrm{m}^{2}$. En parcelas de $10 \mathrm{~m}^{2}$ el número máximo de especies registrado fue de 21 especies (Figura 3A). Los estimadores no-paramétricos en base a las 12 subparcelas de $1 \mathrm{~m}^{2}$ indicaron que la zona de estudio contendría entre 21 y 35 especies de hierbas, arbustos menores y cactáceas (Figura 3B). En el valle del Pampas las parcelas tuvieron diversidades Shannon entre 1.46 bits/individuo (árboles, Pam3) y 2.47 bits/individuo (hierbas, Pam1).

Diversidad y estructura de los BSI del Torobamba.

Dentro de los registros obtenidos en las PMW localizadas en el valle del río Torobamba se encontraron 25 familias, donde las más dominantes fueron Asteraceae con 9 especies y morfoespecies, así como la familia Poaceae con 8 especies y morfoespecies. Encontramos también 63 especies y morfoespecies de las cuales ocho son los árboles Vachellia aroma, Cyathostegia mathewsii, Senegalia sp., Dodonaea viscosa, Sapindus saponaria, Schinus molle, Vasconcellea quercifolia y Eriotheca cf. vargasii. También se registraron 5 suculentas entre ellas Browningia viridis, 16 arbustos, 30 hierbas, 2 helechos y 2 epífitas.

En las cinco parcelas de $400 \mathrm{~m}^{2}$ localizadas en el valle del río Torobamba, se registraron 81 individuos con un DAP $\geq 5 \mathrm{~cm}$ (incluye individuos de tallos múltiples), de los cuales se identificaron 6 especies leñosas o suculentas, siendo en orden decreciente:
Eriotheca cf. vargasii (51 individuos), Ipomoea pauciflora (15 individuos), Vachellia aroma (10 individuos), Vasconcellea quercifolia (3 individuos), Senegalia sp. (1 individuo); se incluye también a Browningia viridis (1 individuo). La especie con mayor DAP en las parcelas de $400 \mathrm{~m}^{2}$ fue Eriotheca cf. vargasii (123.7 cm en la parcela Tor5). En subparcelas de $1 \mathrm{~m}^{2}$ encontramos que la especie Heteropogon contortus fue la especie dominante. En las parcelas de $10 \mathrm{~m}^{2}$ y $100 \mathrm{~m}^{2}$ las especies más abundantes fueron Bidens pilosa y Vachellia aroma respectivamente.

Las curvas de acumulación de especies obtenidas a partir de las 5 parcelas evaluadas dentro del valle del río Torobamba muestran que en parcelas de $400 \mathrm{~m}^{2}$ el número máximo de especies registrado fue de 29 especies. En las parcelas de $100 \mathrm{~m}^{2}$ se registraron 25 especies y en las parcelas de $10 \mathrm{~m}^{2}$ el número máximo de especies registrado fue de 17 especies (Figura 4A). Los estimadores no-paramétricos indican que en base a las 18 subparcelas de $1 \mathrm{~m}^{2}$ la zona de estudio albergaría entre 37 y 47 especies de hierbas, arbustos menores y cactáceas (Figura 4B). Las parcelas en el Torobamba tuvieron diversades Shannon entre 1.69 bits/ individuo (árboles, Tor5) y 2.56 bits/individuo (hierbas, Tor1).

Relaciones florísticas en los BSI del Pampas y Torobamba.

Al analizar las relaciones florísticas de las parcelas de los BSI del valle del río Torobamba y el río Pampas con otras parcelas evaluadas en el sistema de BSI del Apurímac, observamos que las parcelas con la riqueza de especies más alta se encuentran en el Apurímac (Tabla 2). Los demás inventarios tuvieron riquezas entre 4 y 15 especies. El número de especies

Tabla 2. Riqueza de especies (diagonal), especies compartidas (triángulo superior, sombreado claro) y riqueza combinada (triángulo inferior, sombreado oscuro) de inventarios florísticos en el sistema de BSI del Apurímac. Los tres valores más altos para cada caso se resaltan en negrita.

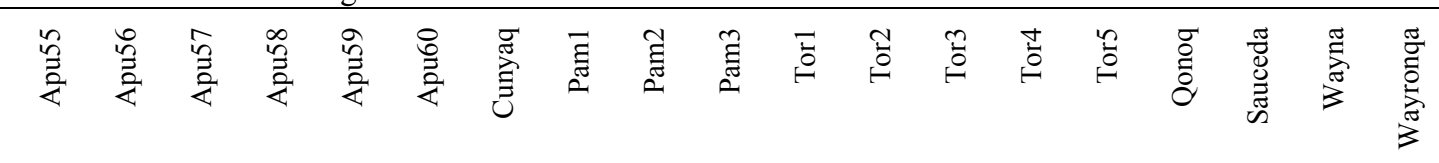

\begin{tabular}{lrrrrrrrrrrrrrrrrrrrrr}
\hline Apu55 & $\mathbf{2 2}$ & 0 & 0 & 0 & $\mathbf{1 1}$ & 2 & 1 & 3 & 3 & 1 & 4 & 4 & 3 & 4 & 3 & 3 & 4 & 0 & 3 \\
Apu56 & 28 & 6 & 0 & 1 & 1 & 0 & 0 & 0 & 0 & 0 & 0 & 0 & 0 & 0 & 0 & 0 & 0 & 0 & 0 \\
Apu57 & 24 & 8 & 2 & 0 & 0 & 0 & 0 & 0 & 0 & 0 & 0 & 0 & 0 & 0 & 0 & 0 & 0 & 0 & 0 \\
Apu58 & 26 & 9 & 6 & 4 & 2 & 2 & 0 & 0 & 0 & 0 & 0 & 0 & 0 & 0 & 0 & 0 & 0 & 0 & 0 \\
Apu59 & $\mathbf{6 3}$ & 57 & 54 & 54 & $\mathbf{5 2}$ & 2 & 1 & 3 & 3 & 1 & 4 & 4 & 3 & 5 & 1 & 3 & 0 & 0 & 3 \\
Apu60 & 30 & 16 & 12 & 12 & 60 & 10 & 0 & 0 & 0 & 0 & 0 & 0 & 0 & 0 & 0 & 0 & 0 & 0 & 0 \\
Cunyaq & 28 & 13 & 9 & 11 & 58 & 17 & 7 & 0 & 1 & 2 & 2 & 0 & 0 & 2 & 1 & 5 & 2 & 4 & 1 \\
Pam1 & 29 & 16 & 12 & 14 & 59 & 20 & 17 & 10 & 2 & 3 & 5 & $\mathbf{6}$ & $\mathbf{6}$ & 5 & 4 & 0 & 0 & 0 & 1 \\
Pam2 & 26 & 13 & 9 & 11 & 56 & 17 & 13 & 15 & 7 & 2 & 3 & 2 & 2 & 5 & 2 & 1 & 1 & 0 & 0 \\
Pam3 & 28 & 13 & 9 & 11 & 58 & 17 & 12 & 14 & 12 & 7 & 3 & 1 & 1 & 3 & 1 & 2 & 1 & 1 & 0 \\
Tor1 & 27 & 15 & 11 & 13 & 57 & 19 & 14 & 14 & 13 & 13 & 9 & $\mathbf{6}$ & $\mathbf{6}$ & $\mathbf{6}$ & 3 & 3 & 1 & 1 & 2 \\
Tor2 & 26 & 14 & 10 & 12 & 56 & 18 & 15 & 12 & 13 & 14 & 11 & 8 & $\mathbf{7}$ & 4 & 2 & 1 & 0 & 0 & 2 \\
Tor3 & 30 & 17 & 13 & 15 & 60 & 21 & 18 & 15 & 16 & 17 & 14 & 12 & 11 & 4 & 2 & 1 & 0 & 0 & 2 \\
Tor4 & 33 & 21 & 17 & 19 & 62 & 25 & 20 & 20 & 17 & 19 & 18 & 19 & 22 & $\mathbf{1 5}$ & 2 & 2 & 1 & 1 & 2 \\
Tor5 & 27 & 14 & 10 & 12 & 59 & 18 & 14 & 14 & 13 & 14 & 14 & 14 & 17 & 21 & 8 & 1 & 2 & 0 & 1 \\
Qonoq & 33 & 20 & 16 & 18 & $\mathbf{6 3}$ & 24 & 16 & 24 & 20 & 19 & 20 & 21 & 24 & 27 & 21 & 14 & $\mathbf{6}$ & 3 & 1 \\
Sauceda & 31 & 19 & 15 & 17 & $\mathbf{6 5}$ & 23 & 18 & 23 & 19 & 19 & 21 & 21 & 24 & 27 & 19 & 21 & 13 & 1 & 0 \\
Wayna & 32 & 16 & 12 & 14 & 62 & 20 & 13 & 20 & 17 & 16 & 18 & 18 & 21 & 24 & 18 & 21 & 22 & 10 & 1 \\
Wayronqa & 28 & 15 & 11 & 13 & 58 & 19 & 15 & 18 & 16 & 16 & 16 & 15 & 18 & 22 & 16 & 22 & 22 & 18 & 9 \\
\hline
\end{tabular}


compartidas entre pares de sitios fue bajo, y sólo un par compartió 11 especies, algunos otros pares compartieron entre 5 y 7 , pero la mayoría entre 1 y 3 especies (Tabla 2). Estas características se reflejan en el dendrograma basado en la disimilaridad entre todos los inventarios del BSI del Apurímac disponibles. Se observaron tres grupos principales de parcelas ubicadas (i) en el valle del Apurímac (altura del cruce del puente Cunyac), (ii) en la confluencia del Pampas con el Apurímac y (iii) en la sección intermedia del Pampas y el valle de su afluente Torobamba (Figura 5). El bajo número de especies compartidas entre grupos se refleja también en los altos valores de disimilaridad que varían entre el $80 \%$ y $90 \%$ (Figura 5).

\section{Discusión.}

Diversidad y estructura de los BSI.

El uso de parcelas de $20 \mathrm{~m}$ x $20 \mathrm{~m}$ en la presente evaluación se justifica en que no se observó un incremento significativo en la cantidad de especies registradas con el aumento del tamaño de las parcelas (Figuras 2 y 3, Tabla 2). La combinación de subparcelas anidadas de distinto tamaño asociado al enfoque en formas de vida particulares acordes al tamaño de la subparcela provee una caracterización adecuada de estos bosques.

Los análisis de estimación de especies a nivel de subparcelas de $1 \mathrm{~m}^{2}$ indican que aún no se ha completado el registro de toda la riqueza de especies presente. Para el Pampas habría aún 4 a 18 especies adicionales por registrar, mientras que en Torobamba se estimaron 5 a 10 especies adicionales. Al considerar la totalidad de las plantas vasculares, y usando la información completa de cada parcela en los dos valles, se estimó entre 14 y 52 especies adicionales por registrar para estos BSI. El desarrollo de inventarios cuantitativos y colectas asistemáticas adicionales en zonas intermedias, enfocándose especialmente en hierbas, arbustos menores y cactáceas, permitiría la detección de las especies adicionales indicadas por cada estimador.

La especie con mayor cobertura en los valles del Pampas y Torobamba fue Heteropogon contortus, mientras que entre los árboles y arbustos las especies más abundantes fueron Eriotheca cf. vargasii y Vachellia aroma, respectivamente. Estos resultados difieren de los registros de Arestegui et al. (2012) para la zona sur de los bosques del Apurímac, donde las especies más abundantes fueron Opuntia ficus-indica (zona Wayronq'a), Furcraea andina (zona Sauceda), Guilleminea densa (zona Wayna) y Vargaseniopsis vargasii (zona Qonqo).

Los resultados indican que el BSI del Pampas (entre 17 a 36 especies por parcela de $0.04 \mathrm{ha})$ y del Torobamba (22 a 29 especies por parcela de 0.04 ha) tienen niveles de riqueza por parcela similares a los reportes para el valle del Marañón donde se reportaron entre 3 a 29 especies por parcela de 0.1 ha (Marcelo-
Peña et al., 2015), pero menores a los registros para el valle del Apurímac donde se reportan 22 a 40 especies por parcela de 0.1 ha (Aréstegui et al., 2012). Por otro lado, la riqueza total obtenida para los BSI del Pampas y del Torobamba es menor en comparación con otros valles secos interandinos como el Marañón donde se reportan 92 especies, 72 géneros y 30 familias (Marcelo-Peña et al., 2015), el Apurímac con valores de 122 especies, 100 géneros y 42 familias (Aréstegui et al., 2012) o los registros de Vargas (1949) de 211 especies, 153 géneros y 43 familias botánicas.

La proporción de las principales formas de vida registradas tanto en los BSI del Pampas (8\% árboles, $15 \%$ arbustos y $58 \%$ hierbas, epifitas $5 \%$, helechos $7 \%$ y suculentas 7\%) como del Torobamba (13\% árboles, $25 \%$ arbustos y $48 \%$ hierbas, epifitas $3 \%$, helechos $3 \%$ y suculentas 8\%), en comparación a los resultados obtenidos por Quintana et al. (2017) (20\% árboles, 36\% hierbas y $44 \%$ arbustos) en los BTES de Ecuador, presentan una menor proporción de árboles y arbustos, y una mayor proporción de especies herbáceas en ambos valles. La proporción de árboles está por debajo
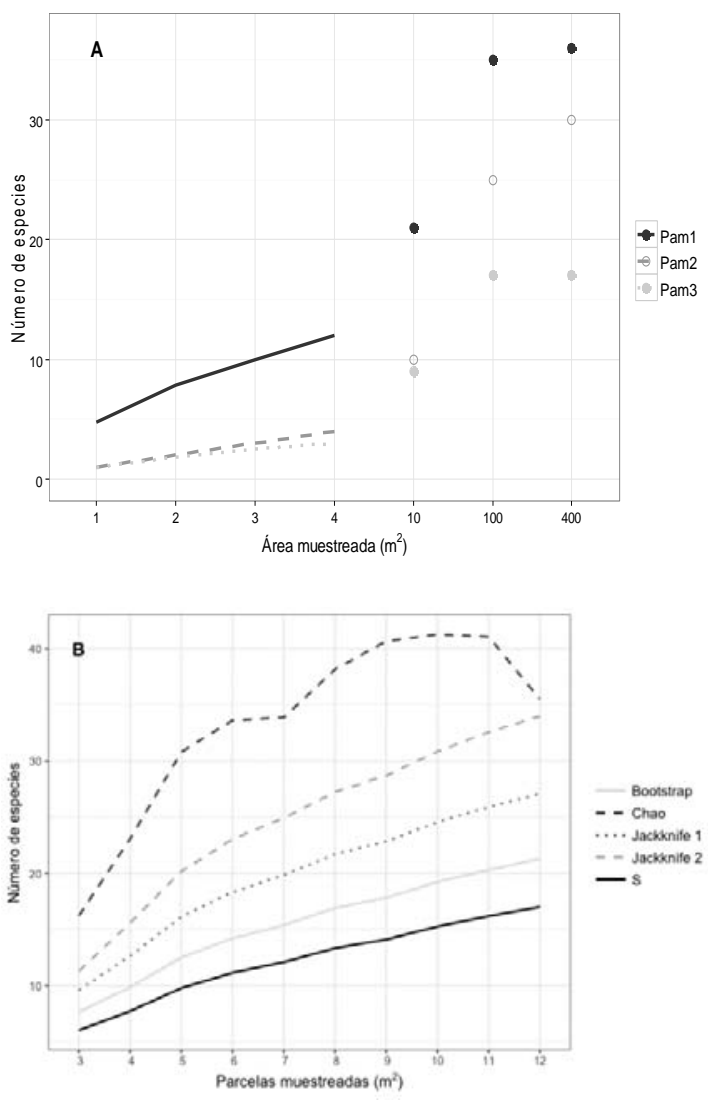

Figura 3. Curvas de acumulación de especies del valle del río Pampas (Tabla 1) en base a tres parcelas y sus subparcelas respectivas (A). Estimación de la riqueza de especies usando parcelas de $1 \mathrm{~m}^{2}$, $\mathrm{S}$ : riqueza de especies observada (B). 
de los resultados obtenidos por Linares-Palomino et al. (2015) (15 a 25\% arboles, 15 a 20\% arbustos y 20 a $30 \%$ herbáceas) para BTES neotropicales. La proporción de arbustos se encuentra dentro de un rango similar, mientras que las herbáceas superarían los registros encontrados para otros BTES, incluso sobrepasando los registros de los bosques de México (56\% de herbáceas, Linares-Palomino et al., 2015). Estos resultados también indicarían que el componente no-arbóreo del bosque de ambos valles contiene un importante porcentaje de la diversidad especifica cómo se ha registrado en otros estudios (Gentry \& Dodson, 1987; Linares-Palomino et al., 2009, 2015).

Relaciones florísticas en los BSI.

Los BSI del río Apurímac y sus tributarios conforman una red compleja de fragmentos, con el componente más representativo y diverso ubicado en el mismo valle del Apurímac entre los 1200 y 2200 msnm. Estos fragmentos de BSI se hacen cada vez más pequeños, aislados y paupérrimos (en términos de diversidad y composición florística) conforme se ubiquen más lejos de este centro de diversidad. Nuestros registros indican que la parcela más densa en herbáceas (Tor2 con $61.5 \%$ de cobertura) se encuentra a mayor altitud (2 $334 \mathrm{msnm}$ ), mientras que la parcela
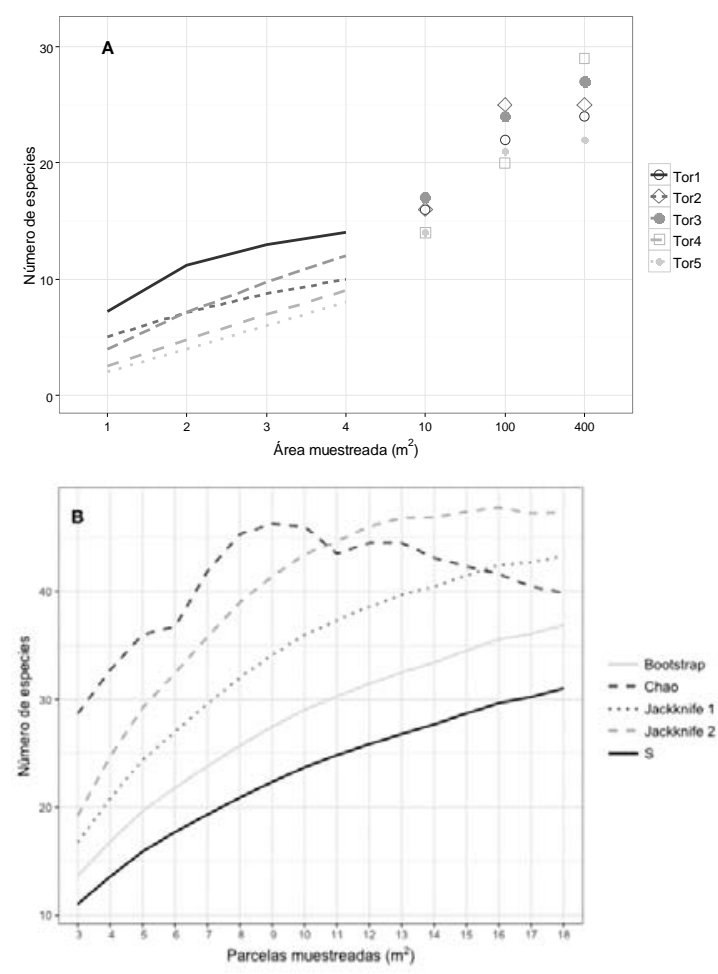

Figura 4. Curvas de acumulación de especies del valle del río Torobamba (Tabla 1) en base a cinco parcelas y sus subparcelas respectivas (A). Estimación de la riqueza de especies usando parcelas de $1 \mathrm{~m}^{2}$, S: riqueza de especies observada (B). más densa en árboles (Pam3 con 30 árboles) es la que está a menor altitud (1845 msnm). Nuestros resultados de diversidad Shannon contrastan con los de Aréstegui et al. (2012), ubicados en el centro de diversidad vegetal del Apurímac, que presentan índices de diversidad de hasta 3.6 bits/individuo a altitudes de 2 859 y 3.3 bits/individuo en la parcela a menor altitud (1 $980 \mathrm{msnm}$ ). Särkinen et al. (2012) mencionan que los biomas de elevación media y alta presentan elevada diversidad de especies y numerosa riqueza específica y de género, mientras los BSI de baja elevación serían menos ricos en géneros y especies, aun cuando todos presentan elevados grados de endemismo.

Estado de conservación de los BSI del sistema Apurímac.

Se identificaron 14 especies en alguna categoría de conservación ( 6 endémicas, 4 amenazadas en Perú y 8 a nivel internacional) lo que corresponde a un $15 \%$ del total de especies registradas. Un 7\% corresponde a endemismos, porcentaje que está por debajo de los registros para el valle del Marañón (33\% y 38\% según datos de Marcelo-Peña et al., 2015 y Linares-Palomino, 2006, respectivamente) que consideran sólo especies arbóreas. Una explicación del bajo nivel de endemismo encontrado en nuestro estudio, podría ser la cantidad de especies que no se lograron identificar $(8 \%$ a nivel de familia, $38 \%$ a nivel de género y $10 \%$ no identificada en ninguna taxa) por la falta de estructuras vegetativas (nuestra expedición se desarrolló durante el pico de la temporada seca). Estudios en los bosques secos del valle del Urubamba, muestran patrones similares con niveles bajos de endemismo aun cuando la mayor parte de la flora ha sido identificada (I. Huamantupa et al., en prensa), lo que indicaría que es una característica de los BSI del sur peruano. Un hecho importante de resaltar es que es necesario realizar inventarios de plantas durante la época de lluvias, que en estos ecosistemas se restringe debido a la severidad de las mismas (LinaresPalomino et al., 2015; Killeen et al., 2008).

Los resultados que presentamos dejan claro que no será suficiente asegurar la protección de estos bosques concentrándonos sólo en las zonas con mayor diversidad (Fleishman et al., 2006). Por el contrario, se hace evidente que una red de áreas de conservación a lo largo de este valle y sus tributarios tendrían las mayores posibilidades de conservar una muestra representativa de los bosques secos interandinos del Apurímac.

La presente evaluación es uno de los primeros estudios florísticos de plantas vasculares para los valles interandinos del río Pampas y Torobamba. Queda pendiente mejorar la base cartográfica de estos bosques para conocer el tamaño, la ubicación, la calidad de los remanentes, y su estado de regeneración. El estado actual y las amenazas a las que están expuestos estos bosques no es diferente de la realidad de otros BTES en el Perú (e.g. Marcelo-Peña et al., 2015) e incluyen la falta de áreas naturales protegidas, fragmentación del paisaje, cambio de uso de tierra y presencia de ganado. 


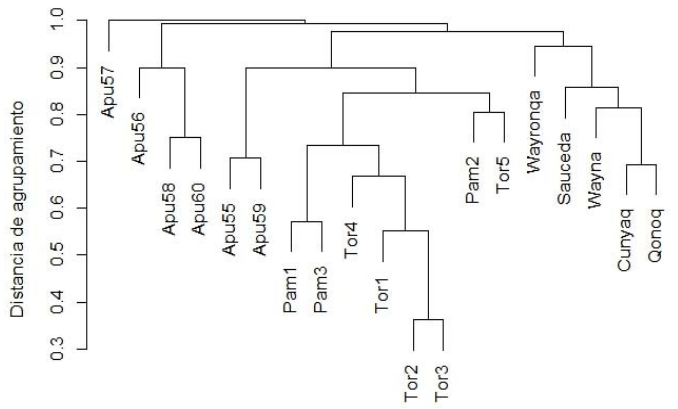

Figura 5. Análisis de agrupamiento UPGMA, basado en matriz de disimilaridad de Bray-Curtis de las parcelas del Apurímac y Cusco (Aréstegui et al., 2012; K Dexter, datos no publicados) y del valle del río Pampas y Torobamba (Tabla 1).

Todos estos factores contribuyen a la degradación de estos bosques que en zonas como Torobamba ya están parcialmente dominados por el arbusto Dodonaea viscosa (Sapindaceae), una especie exótica reconocida como una planta invasora en bosques estacionales del Brasil (Ziller et al., 2005). La baja densidad de plantas juveniles y recién germinadas de especies características como E. cf. vargasii que encontramos en este estudio nos sugiere que la regeneración de estos bosques se da de manera extremadamente lenta. Todas estas condiciones hacen urgente el desarrollo de estudios básicos y aplicados que contribuyan a un mejor entendimiento del funcionamiento de estos bosques y a plantear las opciones de manejo más adecuadas para su preservación y la de los servicios ecosistémicos que brindan a las poblaciones aledañas.

\section{Agradecimientos.}

Este estudio fue posible gracias al apoyo financiero y logístico de la empresa PERU LNG como parte del Programa de Monitoreo y Evaluación de la Biodiversidad (BMAP, por sus siglas en inglés), una colaboración entre PERU LNG y el SCBI. Este estudio se realizó bajo la autorización de investigación 01442012-AG-DGFFS-DGEFFS. Agradecemos a Kyle Dexter (Universidad de Edimburgo) por proporcionarnos datos no publicados de sus inventarios en el Apurímac. Agradecemos al Herbario USM del Museo de Historia Natural de la Universidad Nacional Mayor de San Marcos por permitirnos el uso de sus instalaciones y a María Isabel La Torre por su apoyo en la identificación de muestras. Esta es la contribución No. 54 del Programa de Biodiversidad del Perú del Smithsonian Conservation Biology Institute.

\section{Literatura citada.}

Aréstegui A., Tupayachi A., Acurio L., Yabarrena J.E., Zamalloa V. \& Farfán J. 2012. Flora xerofítica de la Cuenca de Apurímac: Limatambo-Curahuasi. Revista Universitaria. 141: 95-108.

Barnett D.T. \& Stohlgren T.J. 2003. A nested-intensity design for surveying plant diversity. Biodiversity and Conservation. 12: 255-278.

DRYFLOR, Banda-R K., Delgado-Salinas A., Dexter K.G., Linares-Palomino R., Oliveira-Filho A., Prado D., Pullan M., Quintana C., Riina R., Rodríguez M.G., Weintritt J., Acevedo-Rodríguez P., Adarve J., Álvarez E., Aranguren B.A., Arteaga J.C., Aymard G., Castaño A., CeballosMago N., Cogollo A., Cuadros H., Delgado F., Devia W., Dueñas H., Fajardo L., Fernández A., Fernández M.A., Franklin J., Freid E.H., Galetti L.A., Gonto R., GonzálezM. R., Graveson R., Helmer E.H., Idárraga A., López R., Marcano-Vega H., Martínez O.G., Maturo H.M., McDonald M., McLaren K., Melo O., Mijares F., Mogni V., Molina D., del Pilar Moreno N., Nassar J.M, Neves D.M., Oakley L.J., Oatham M., Olvera-Luna A.R., Pezzini F.F., Reyes Dominguez O.J., Ríos M.E., Rivera O., Rodríguez N., Rojas A., Särkinen T., Sánchez R., Smith M., Vargas C., Villanueva B. \& Pennington R.T. 2016. Plant diversity patterns in neotropical dry forests and their conservation implications. Science. 353: 13831387.

Fleishman E., Noss R.F. \& Noon B.R. 2006. Utility and limitations of species richness metrics for conservation planning. Ecological Indicators. 6: 543-553.

Gagnon E., Hughes C.E., Lewis G.P. \& Bruneau A. 2015. A new cryptic species in a new cryptic genus in the Caesalpinia group (Leguminosae) from the seasonally dry inter-Andean valleys of South America. Taxon. 64: 468-490.

Gentry A.H. \& Dodson C. 1987. Contribution of nontrees to species richness of a tropical rain forest. Biotropica. 19: 149-156.

Hammer Ø., Harper D.A.T., Ryan P.D. 2001. PAST: Paleontological statistics software package for education and data analysis. Palaeontologia Electronica. 4: 1- 9.

Huamantupa I. \& Cuba M. 2013. Plantas de Limatambo, Cunyac y Curahuasi, Bosque Seco Inter-Andino, Departamentos Cusco-Apurímac, Peru. Disponible en http://fieldguides.fieldmuseum.org/guides/guide/469 Luza-Victorio M., Mollepaza E. \& Linares-Palomino R. En prensa. Woody plant diversity in the seasonally dry tropical forests of the Urubamba valley, a threatened biodiversity hotspot in southern Peru. Tropical Ecology.

IUCN. 2013. IUCN Red List of Threatened Species. Version 2013.2. Disponible en: http://www.iucnredlist.org

Killeen T.J., Douglas M., Consiglio T., Jorgensen P.M. \& Mejia J. 2008. Dry spots and wet spots in the Andean hotspots. Journal of Biogeography. 34: 1357-1373.

Linares-Palomino R. 2004. Los Bosques Tropicales Estacionalmente Secos: II. Fitogeografía y Composición florística. Arnaldoa. 11:103-138.

2006. Phytogeography and floristics of seasonally dry forests in Peru. Páginas 257-279. En: R.T. Pennington, G.P. Lewis \& J.A. Ratter (editores) Neotropical Savannas and Seasonally Dry Forests: Plant 
Diversity, Biogeography and Conservation. CRC, Boca Raton, FL.

2013. Plant diversity, composition and structure of the seasonally dry forest in Torobamba, an inter-Andean valley in Ayacucho, southern Peru. Setting the baseline for long-term ecological monitoring and conservation of the endemic tree Eriotheca vargasii. Páginas 41-51. En: A. Alonso, F. Dallmeier \& G.P. Servat (editores) Monitoring Biodiversity: lessons from a TransAndean Megaproject. Smithsonian Institution Scholarly Press.

2016. Plantas vasculares de los bosques secos del Torobamba y Pampas, Ayacucho, Perú. Disponible http://fieldguides.fieldmuseum.org/guides/guide/735 , Cardona V., Hennig E.I., Hensen I., Hoffmann D., Lendzion J., Soto D., Herzog S.K. \& Kessler M. 2009. Non-woody life-form contribution to vascular plant species richness in a tropical American forest. Plant Ecology. 201: 87-99. , Aybar D. \& Morales E. 2015. Floristics of neotropical seasonally dry forests: a perspective from complete vascular plant inventories. Phytocoenologia. 45: 251-267.

Magurran, A. 2004. Measuring Biological Diversity. Blackwell Science Ltd. 100-130.

Marcelo-Peña J.L., Huamantupa I., Särkinen T. \& Tomazello M. 2015. Identifying conservation priority areas in the Marañón valley (Peru) based on floristic inventories. Edinburgh Journal of Botany. 73: 1-29.

Miles L., Newton A.C., DeFries R.S., Ravilious C., May I., Blyth S., Kapos V. \& Gordon J.E. 2006. A global overview of the conservation status of tropical dry forests. Journal of Biogeography. 33: 491-505.

Morlon H., Chuyong G., Condit R., Hubbell S., Kenfack D., Thomas D., Valencia R. \& Green J.L. 2008. A general framework for the distance-decay of similarity in ecological communities. Ecology letters. 11:904-917.

Nekola J.C. \& White P.S. 1999. The distance decay of similarity in biogeography and ecology. Journal of Biogeography. 26: 867-878.

Oksanen J., Blanchet F.G., Kindt R., Legendre P., Minchin P.R., O'Hara R.B., Simpson G.L., Solymos P., Stevens M.H.H. \& Wagner H. 2016. Vegan: Community Ecology
Package. R package version 2.3-4. Disponible en http://CRAN.R-project.org/package=vegan

Portillo-Quintero C.A. \& Sánchez-Azofeifa G.A. 2010. Extent and conservation of tropical dry forests in the Americas. Biological Conservation. 143: 44-155.

Quintana C., Girardello M., Barfod A.S. \& Balslev H. 2017. Diversity patterns, environmental drivers and changes in vegetation composition in dry inter-Andean valleys. Journal of Plant Ecology. 10: 461-475.

R Core Team. 2014. R: A language and environment for statistical computing. R Foundation for Statistical Computing, Vienna, Austria. Disponible en: http://www.R-project.org/

Rauh W. 1958. Beitrag zur Kenntniss der Peruanischen Kakteenvegetation. Sitzungsberichte der Heidelberger Akademie der Wissenschaften, MathematischeNaturwissenschaftliche Klasse. 1-542.

Sánchez-Azofeifa G.A., Kalacska M., Quesada M., CalvoAlvarado J.C., Nassar J.M. \& Rodríguez J.P. 2005. Need for integrated research for a sustainable future in tropical dry forests. Conservation Biology. 19: 285-286.

Särkinen T.E., Marcelo-Peña J.L., Yomona A.D., Simon M.F., Pennington R.T. \& Hughes C.E. 2011. Underestimated endemic species diversity in the dry inter-Andean valley of the Río Marañón, northern Peru: An example from Mimosa (Leguminosae, Mimosoideae). Taxon. 60: 139-150.

, Pennington R.T., Lavin M., Simon M.F. \& Hughes C.E. 2012. Evolutionary islands in the Andes: persistence and isolation explain high endemism in Andean dry tropical forests. Journal of Biogeography. 39: 884-900.

UNEP-WCMC (Comps.). 2015. The Checklist of CITES Species Website. CITES Secretariat, Geneva, Switzerland. Compiled by UNEP-WCMC, Cambridge, UK. Disponible en: $\mathrm{http}: / /$ checklist.cites.org

Vargas C. 1949. La flora xerofita del Apurímac medio. Lilloa. 20: $215-236$

Weberbauer A. 1945. El mundo vegetal de los Andes Peruanos: Estudio fitogeográfico. Segunda edición. Ministerio de Agricultura. Lima. Perú.

Ziller S.R., Reaser J.K., Neville L.E. \& Brandt K. 2005. Invasive alien species in South America: national reports $\&$ directory of resources. Global Invasive Species Programme, Cape Town, South Africa.

Anexo 1. Lista de especies registradas en los valles secos del río Torobamba y Pampas. No incluye 7 hierbas, 2 helechos y un arbusto que no pudieron ser identificados por falta de material fértil.

\begin{tabular}{|c|c|}
\hline Taxón registrado & $\begin{array}{l}\text { Forma de } \\
\text { crecimiento }\end{array}$ \\
\hline \multirow{2}{*}{\multicolumn{2}{|c|}{$\begin{array}{l}\text { CLASE MAGNOLIOPSIDA } \\
\text { FAMILIA AMARANTHACEAE }\end{array}$}} \\
\hline & \\
\hline Alternanthera sp. & Hierba \\
\hline $\begin{array}{l}\text { Iresine diffusa Humb. \& Bonpl. ex Willd. } \\
\text { FAMILIA ANACARDIACEAE }\end{array}$ & Hierba \\
\hline $\begin{array}{l}\text { Schinus molle L. } \\
\text { FAMILIA APOCYNACEAE }\end{array}$ & Árbol \\
\hline $\begin{array}{l}\text { Asclepias curassavica L. } \\
\text { FAMILIA ARALIACEAE }\end{array}$ & Hierba \\
\hline $\begin{array}{l}\text { 5. } \\
\text { Aralia soratensis Marchal } \\
\text { FAMILIA ARISTOLOCHIACEAE }\end{array}$ & Arbusto \\
\hline Aristolochia sp. & Hierba \\
\hline
\end{tabular}


Anexo 1. Lista de especies registradas en los valles secos del río Torobamba y Pampas. No incluye 7 hierbas, 2 helechos y un arbusto que no pudieron ser identificados por falta de material fértil.

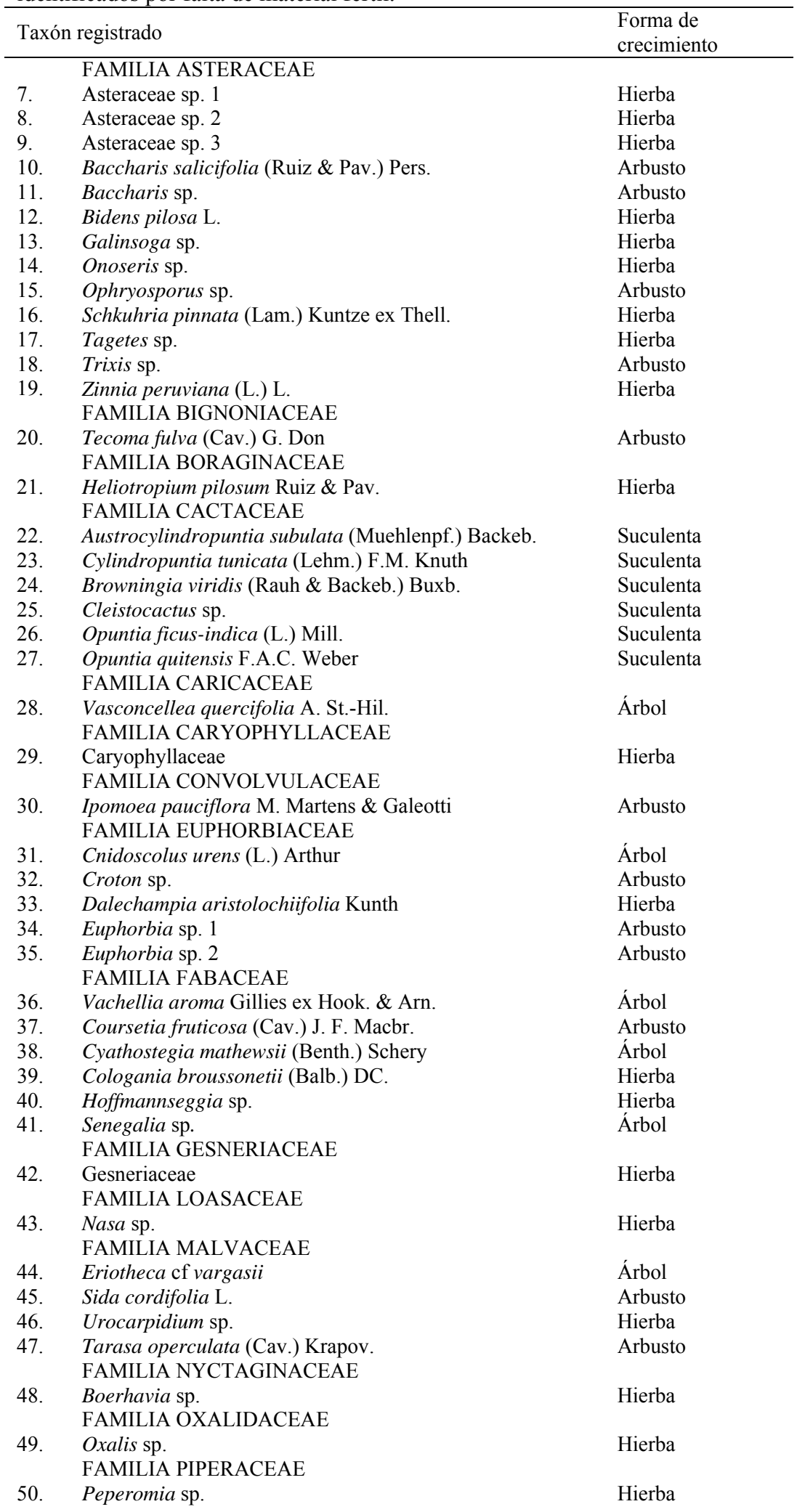


Anexo 1. Lista de especies registradas en los valles secos del río Torobamba y Pampas. No incluye 7 hierbas, 2 helechos y un arbusto que no pudieron ser identificados por falta de material fértil.

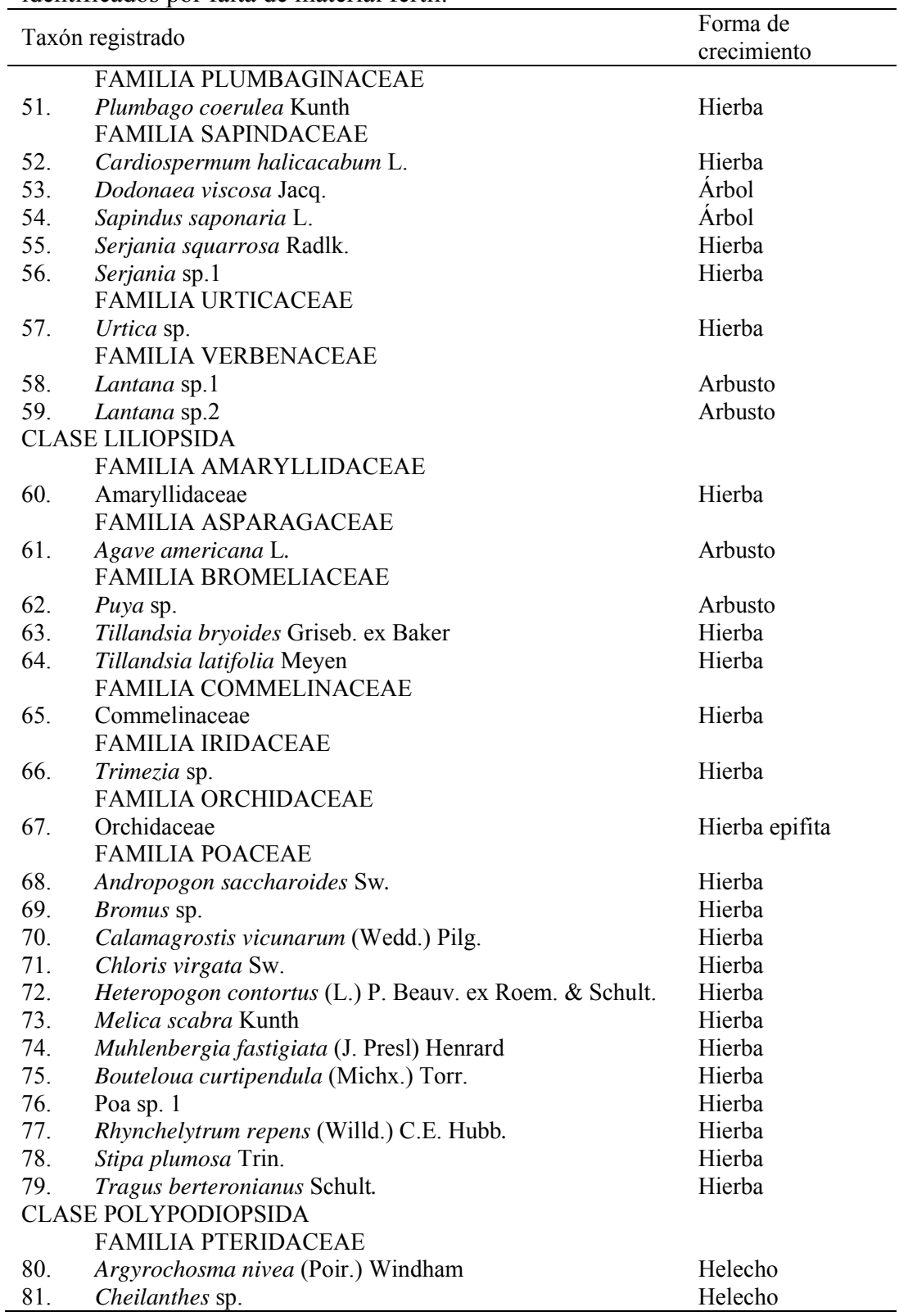

${ }^{1}$ Smithsonian Conservation Biology Institute, Los Libertadores 215, San Isidro, Lima \& Agropecuaria ARZU, Calle Ilo 882, Moquegua, fioedupa@gmail.com.

${ }^{2}$ Smithsonian Conservation Biology Institute, Los Libertadores 215, San Isidro, Lima. hchuquillanqui@gmail.com.

${ }^{3}$ Asociación Pro Fauna Silvestre, Jr. José Santos Flores, Mz. A1 Lt. 9 Las Américas, San Juan Bautista, Ayacucho \& Instituto de Investigación en Biodiversidad y Ecosistemas Altoandinos, Av. Independencia S/N Ciudad Universitaria, Ayacucho.napace16@gmail.com.

${ }^{4}$ Smithsonian Conservation Biology Institute, Los Libertadores 215, San Isidro, Lima. linaresr@si.edu. 